\title{
THE ANTIBIOTICS IN ABDOMINAL INFECTIONS
}

\author{
By Ivor LewIS, M.D., M.S.(Lond.), F.R.C.S.(Eng.) \\ St. Asaph, North Wales
}

The mortality of acute abdominal infections has greatly fallen in the last 15 or 20 years. Acute appendicitis for example had an overall mortality 20 years ago of about 5 per cent., whereas now it is probably rather less than I per cent. A similar improvement has occurred in perforated peptic ulcer. It is obvious that there are several factors responsible for this improvement, e.g. timely surgery, natural fluctuation in the virulence and prevalence of infections, but the use of the antibiotics has certainly played an important part. The response of cases of fulminating peritonitis, for example, is more impressive to surgeons who remember these cases 20 years ago, than any statistics can be. The sulphonamides had already done something to lower the mortality of such cases. It will be convenient to summarize the use of the antibiotics in this connection under three headings: By themselves, post-operatively and preoperatively.

\section{Antibiotics by Themselves}

Conditions suitable for this line of treatment form a small but important group, almost entirely cases of general peritonitis-sometimes with, but usually without, a causal focus.

Primary pneumococcal peritonitis is best treated with antibiotics alone; penicillin in doses of, say, roo, 000 units four-hourly. Similarly primary streptococcal peritonitis. In both conditions a specimen is taken for culture with a very fine needle, so that subsequent treatment can be guided by bacteriological sensitivity. Occasionally residual collections may need drainage.

Gonococcal peritonitis, arising from salpingitis, is nowadays seldom seen, but is abruptly cut short by a few days' penicillin therapy.

Tubercular peritonitis, both the wet and dry varieties, usually responds well to streptomycin in doses of $2 \mathrm{~g}$. alt. die plus PAS $18 \mathrm{~g}$. daily. This is continued for two or three months, depending of course on the presence or absence of other lesions.

In all these conditions antibiotics have transformed the treatment. No operation is required except where necessary to establish the diagnosis and, now and again, to drain a residual collection of pus. Finally, under this heading must be mentioned the occasional case of peritonitis, especially in old people, where the exact aetiology is not known-probably a perforated peptic ulcer or stercoral ulcer or maybe a neglected appendicitis, and where the patient is not fit for laparotomy. A number of such cases are now successfully treated by antibiotics plus continuous gastric suctionthe final diagnosis perhaps never becoming known.

\section{Post-operative Use}

This includes the instillation of powder or solution during operations in contaminated or threatened areas of the field.

Local use. It is a common practice to sprinkle a million units of penicillin \pm a gramme of streptos. mycin where the alimentary lumen has ruptured of has been opened during the operation.' Though this has perhaps degenerated into an automatic ritual, nevertheless there is no other method which will bring such a high antibiotic concentration in contact with dangerous organisms so early and so effectively. In the case of continued leakage its value is small. For antibiotics are no substitute for sound surgery; they merely supplement it. Where the colon has been opened during the operation - the acutely obstructed small intestine is even more septic - the utmost care must be practised, as in the past, to reduce contamination to the minimum. If, in addition, penicillin and streptomycin are used locally, a great fall in morbidity will result, with far fewer infections of the wound. But mortality is probably little affected, provided the surgical technique has been good.

In operations specially liable to heavy septic N contamination, such as resection for strangulated hernia or perforated stercoral ulcer, it is still worth emphasizing the value of drainage and $\mathbb{\omega}$ packing of the wound-or at any rate the superficial layers thereof. These measures are used in addition to antibiotics.

It is probable that acutely obstructed gut, after $\stackrel{\oplus}{?}$ handling, will allow organisms to permeate into ${ }^{\circ}$ the peritoneum. Local application of antibiotics seems ideal to meet such a contingency. 
Systemic use. This is beneficial in cases where the infection had already spread beyond the organ of origin, e.g. the appendix. It is only called for where the peritoneal fluid is frankly purulent. We may well take appendicitis as the type of such conditions. The best criterion is probably the smell of the pus. Where there is offensive peritonitis, or where localized perforation has occurred with a black gangrenous appendix wall, it is wise to continue systemic antibiotics for some days. My own practice is to use streptomycin, I g. twice daily, for three or four days. By this time the patient is usually over the worst; in any case the coliform organisms will have become resistant. Some surgeons use penicillin for appendix peritonitis, claiming that there is a synergistic effect: by abolishing the intestinal cocci the coliform organisms are controlled at the same time. Both antibiotics can be used simultaneously; the penicillin will at any rate ward off some coccal infections of the chest. After operation for perforated peptic ulcer where contamination has been severe and lasted over six hours, penicillin should be given -crystalline, 100,000 units four-hourly for three to five days. This will reduce both the peritonitis and pulmonary infection. It is now common to find the mortality of these cases for all ages, kept below 5 per cent., whereas in the 'thirties it was around 20 per cent. in nearly all large centres.

Next to the peritonitis itself pulmonary complications are the commonest cause of death in abdominal infections. Penicillin-or aureomycin in special cases-greatly reduces the incidence and severity. It must be stressed that two of the most serious pulmonary complications are largely preventable: aspiration bronchopneumonia and massive collapse. But antibiotics are a most potent accessory means of reducing the danger. Urinary infections are very common after abdominal operations of all sorts, from prostatectomy to herniorrhaphy. Here again the important thing is prevention as far as possible. Urinary retention can easily be missed in women, unless one always bears in mind that the only common cause of postoperative frequency in women is retention. If the retention is overlooked then sepsis is very liable to follow repeated catheterization.

Provided the bladder is acting normally, the best methods of treatment are ammonium mandelate, sulfadiazine, streptomycin or chloramphenicol-in that order; the use of the last two being guided by laboratory sensitivity.

It is well to remember the value of intermitting the various forms of treatment. Often the eventual sterilization of the urine is abrupt and apparently unrelated to treatment.

\section{Pre-operative Use}

Much has been written about the use of the antibiotics and sulphonamides in the pre-operative preparation for colon resection, but nothing is sounder than the following words of Coller's:

'Pre-operative bowel sterilization with antibiotics has perhaps lowered the morbidity rate and probably the mortality rate of colon surgery, but if the patient is in good nutritional balance and the established principles of bowel surgery are carefully followed, its value is negligible.'

'An anastomosis does not heal because it is $\mathrm{Hi}^{\mathrm{i}}$ a sterile field but because it has been done with minimum trauma, has a good blood supply, and is not under undue tension.'

In the hands of those doing much of this work there had been a large fall in the mortality of colon resections, even before antibiotics came in. But their advent plus the careful technique previously evolved have between them resulted in a reduction in mortality from about 15 per cent. to well below 5 per cent. Most impressive of all has been the lessened incidence of the septic complicationsboth in the peritoneum and in the wound. The pre-operative preparation takes about five days in the absence of obstruction. In this country the commonest method is $8 \mathrm{~g}$. of sulphasuxidine dails for five days. Alternatively sulfathalidine may be used; the latter also acts as a comfortable laxative을

Lately some American surgeons, especially Poth, have pointed out the superiority of neomycin combined with sulfathalidine, for the purpose, as a more complete sterility of the colon can thus be produced than by any other means. This antibiotic is given by the mouth, I g. hourly for four doses then I $g$. four-hourly for four doses, combined with sulfasuxidine. Only yeasts will usually survive. This antibiotic might well be reserved for this pre-operative purpose alone so as to avoid resistant strains developing in colons generally.

Where neomycin is not available for rapid preoperative preparation then streptomycin by the mouth, I g. I2-hourly for three or four doses, will do much to reduce the septic content of the gut.

Chlortetracyclin, oxytetracyclin and tetracyclin are all of considerable effect in sterilizing the gut contents, but the danger of 'staphylococcal cholera' due to resistant strains of staphylococcus outgrowing all else with fulminating rapidity, rules out their use unless there is some very special reason. Erythromycin is effective but resistance very quickly develops. It may be of value where there is strong laboratory support in a particular case.

It must again be emphasized that in colonic obstruction the antibiotics do not replace colostomy 
as the soundest safety measure in most cases, but they are a useful adjunct.

\section{Pre-operative Use in Peritonitis}

Not infrequently cases admitted with a severe peritonitis-most commonly due to appendicitis, are best treated by a 12 hours' pre-operative course of antibiotics, plus continuous gastric suction and intravenous saline $(\mathrm{N} / 5)$. Streptomycin and penicillin are usually advocated, though personally I give streptomycin alone in most cases. The operation can then be performed with a mortality many times lower than before the days of antibiotics. Indeed as for appendicitis, I would say that the case for 'expectant treatment' at any stage has been demolished by the antibiotics. The same valuable indication applies to any case of severe peritonitis of uncertain origin-a few hours' preparation may render an operation possible without undue hazard.

\section{Complications}

By now it is recognized that these are common enough and occasionally dangerous enough to be mentioned whenever antibiotic treatment is discussed. The allergic and toxic complications may vary from rashes (measly or scarlatiniform) to pyrexia and even sudden death from anaphylactic shock. All the above may occur even with aqueous penicillin - thought at one time to be the safest of $c$ drugs. The long-acting compounds of penicillin are likelier to cause these reactions.

It is believed that polyarteritis nodosa and diffuse lupus erythematosus have arisen as complications. Eighth nerve damage due to streptomycin is well known. Both chloramphenicol and streptomycin have been known to cause aplastic \& anaemia, agranulocytosis and thrombocytopenia, which may result from the use of the "widespectrum'drugs such as terramycin and aureomycin. Resistant staphylococci may multiply, causing fulminating staphylococcal enterocolitis, often fatal in two or three days. In other cases yeasts alone may overrun the whole alimentary canal. It is to be noted that the lighthearted and widespread use of penicillin in the last ten years has resulted in only 25 per cent. of human pathogenic staphylococci now being sensitive to penicillin, whereas in 1945 the figure was 85 per 의 cent. Such a fact should condemn the 'routine' administration of antibiotics, a senseless practice $\vec{z}$ which has become so prevalent, not only in hospitals but in outside medical practice.

\section{ADRENAL GLANDS}

(Postgraduate Medical Journal)

Price 3s. 8d. post free

THE ADRENAL STEROIDS

R. I. S. Bayliss, M.A., M.D., M.R.C.P. CUSHING'S SYNDROME

A. C. Crooke, M.A., M.D.

THE ADRENO-GENITAL SYNDROME

S. Leonard Simpson, M.A., M.D., F.R.C.P.

\section{HYPOPITUITARISM}

A. A. G. Lewis, B.Sc., M.D., M.R.C.P.
ADDISON'S DISEASE

G. A. SMART, B.Sc., M.D., F.R.C.P.

SURGERY OF THE ADRENAL GLAND

Selwyn Taylor, M.Ch., F.R.C.S.

ACUTE ADRENAL INSUFFICIENCY

Paul Fourman, M.D., M.R.C.P.

Published by

THE FELLOWSHIP OF POSTGRADUATE MEDICINE

60, Portland Place, London, W.1 